\title{
Synergetic Antibacterial Effects of Silver Nanoparticles@Aloe Vera Prepared via a Green Method
}

\author{
Yixia Zhang*, Dapeng Yang*, Yifei Kong, Xiansong Wang, Omar Pandoli, Guo Gao \\ Department of Bio-Nano-Science and Engineering, National Key Laboratory of Nano/Micro Fabrication Technology, Key Laboratory for Thin Film \\ and Microfabrication of Ministry of Education, Institute of Micro-Nano Science and Technology, Shanghai Jiao Tong University, 800 Dongchuan \\ Road, Shanghai 200240, P.R. China. \\ *Corresponding authors: boyydp@sjtu.edu.cn (D.P. Yang) and shsusan@sjtu.edu.cn(Y.X. Zhang)
}

\begin{abstract}
Aloe Vera-conjugated Ag nanoparticles (AgNPs@AV hybrids) are synthesized in large quantities by reducing silver nitrate with Aloe Vera pulp extract at room temperature. TEM image reveals that these NPs are predominantly spherical with an average of $25 \mathrm{~nm}$ in diameter. The crystal structure of AgNPs@AV is determined by XRD. The cytotoxicity of AgNPs@AV hybrids is detected by carrying out the cell viability measurement on Human Dermal Fibroblasts (HDF) cells, the results show that no obvious cytotoxicity is observed. Compared with Vera gel and Ag NPs (washed from Vera gel) alone,AgNPs@AV hybrids possess more excellent antibacterial activity on E. coli even at very low concentration.
\end{abstract}

Key Words: Silver NPs, Aloe Vera, Synergetic effect, Green synthesis, Cytotoxicity

Citation: Y. Zhang,et al. Synergetic Antibacterial Effects of Silver Nanoparticles@Aloe Vera Prepared via a Green Method. Nano Biomed. Eng. 2010, 2(4), 252-257.DOI: 10.5101/nbe.v2i4.p252-257.

\section{Introduction}

With the development of nanotechnology, controllable synthesis of noble metal NPs has attracted much attention due to their potential applications in many areas [1]. Especially, they have been extensively exploited for use in biomedical areas, such as targeted drug delivery [2], imaging [3], sensing [4] and antimicrobial [5]. Among these metal NPs, Ag NPs are particular interest due to its strong and wide-spectrum antimicrobial activities [6-8], which might act as a novel bactericide to solve the serious antibiotic resistance problem. Ag NPs can be successfully synthesized by traditionally chemical and physical methods. However, these methods strongly depend on severe reaction conditions, for example, aggressive agents (sodium borohydride, hydrazinium hydroxide, cetyltriethylammnonium bromide), harmful solvent system to environment and ecology, higher temperature and higher pressure, and so on. To pursue a healthy life and space, it is imperative to develop a clean synthetic approach ("green chemistry") to obtain nanomaterials targeted on different applications, especially in biomedical fields.
An environmentally acceptable solvent system, ecofriendly reducing and capping agents are considered to be three essential elements for a completely "green" synthesis [9]. Biotemplated strategies might be alternative to achieve the three standards, [10-14] which are based on biological molecules, microorganism or plant extract for synthesis of NPs, such as Au NPs [15], Ag NPs [16] and other functional materials [17, 18]. There are many biologically active components in the extracts of fresh plant, such as proteins, polysaccharides, vitamins, polyphenols and so on. On one hand, some molecules can act as electron shuttles in metal reduction; On the other hand, some constituents are responsible for the capping of resulting NPs. As a result, not only the aggregation of NPs could be effectively avoided, but also the post-surface modification of NPS might be easily completed. Additionally, green plants are reproducible sources which fit for the requirements of sustainable development. Meanwhile, S. Shiv Shankar [19] and co-workers have demonstrated that use of plants extract outweigh microorganisms in the biosynthesis 
of metal NPs. Therefore, the approach based on green plants extract is an intrinsically green alternative to NPs synthesis. In recent years, some environmentally friendly methods based on plants extracts are largely explored. For example, Mallikarjuna [20] and co-workers generated $\mathrm{Ag}$ and Pd NPs at room temperature employing coffee and tea extract. The single-pot method used no surfactant, capping agent, and/or template, which is an intrinsically green approach. Stimulated by these novel synthesis ideas, herein, we attempted to use Aloe Vera extract to synthesize Ag NPs. Aloe Vera leaf contains many biological activity components, including vitamins, enzymes, sugars, salicylic acids, and amino acids so on [21]. As a kind of important natural medical plants which has been reported mostly in clinical medicine [22-24], the pharmacological activities of Aloe Vera, as studied in vitro or in animals (in most cases the total leaf extract was used) including anti-inflammatory [25] anti-arthritic activity [26] and antibacterial effects [27].

In this paper, we report a low-cost, convenient, green synthesis approach to obtain large quantities of AgNPs@ AV by reduction of silver ions with Vera extract at room-temperature. The material exhibited excellent biocompatibility and antibacterial characteristics to E. coli.

\section{Materials and methods}

\subsection{Aloe Vera Extract Preparation}

A $30 \mathrm{~g}$ portion of thoroughly washed Aloe Vera leaves were finely cut and boiled in $100 \mathrm{~mL}$ of sterile distilled water. The resulting extract was used for further experiments. Figure 1 is the optical picture of Aloe Vera used in our experiment.

\subsection{Synthesis of Ag NPs@AV}

For the synthesis of the Ag NPs@AV, we adopted

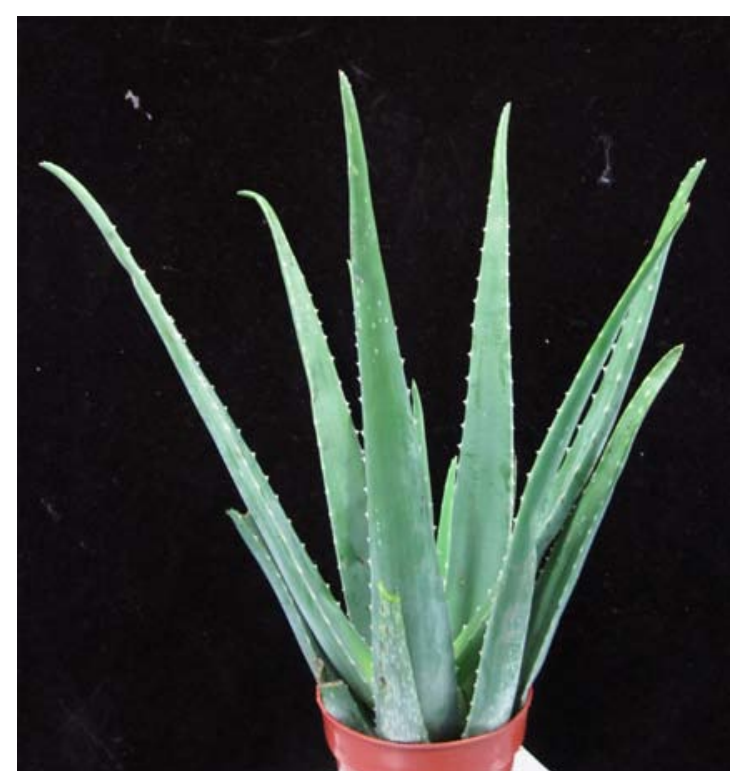

Figure 1 the optical picture of original Aloe Vera previous report [28] with slight modification. Typically, 5 $\mathrm{mL} 10 \mathrm{molL}^{-1} \mathrm{AgNO}_{3}$ solution was added to $5 \mathrm{~mL}$ of Vera extract. The observation of faint yellow color after $24 \mathrm{~h}$ of reaction (avoid light) indicated the formation of $\mathrm{Ag}$ NPs@AV which was further characterized by XRD and TEM measurements.

\subsection{Transmission Electron Microscopy (TEM) measurement and X-Ray study}

TEM samples of the NPs synthesized using the Aloe Vera extract were prepared by placing drops of the reaction mixture over carbon-coated copper grids and allowing the solvent to evaporate. Then TEM measurements were performed on a JEOL model 1200EX instrument operated at an accelerating voltage of 80 $\mathrm{kV}$. The crystalline nature of AgNPs@AV hybrids was confirmed from the X-ray diffraction analysis.

\subsection{Bactericidal effect}

E. Coli was grown in liquid LB medium at $37^{\circ} \mathrm{C}$, overnight, on a shaker up to the stationary growth phase. 1:100 ratio of this culture and LB medium was grown in fresh LB medium until mid-log phase cells $(\mathrm{OD}=0.6)$. Exponential growing cells were centrifuged and suspended in $0.9 \%$ sterilized chloride sodium solution and adjusted to $10^{3} \sim 10^{4} \mathrm{CFU} \mathrm{mL} \mathrm{m}^{-1}$ for further experiment. To examine the antibacterial effect of the NPs on Gramnegative bacteria, approximately $10^{5}$ colony forming units (CFU) of E. coli strain B were cultured on LB agar plates supplemented with AgNPs@AV hybrids in concentrations of $10 \mu \mathrm{molL}^{-1}$ to $500 \mu \mathrm{molL}^{-1}$.AgNPs@AV-free LB plates cultured under the same conditions were used as a control. The counts on the three plates corresponding to a particular sample were averaged. The colonies numbers were counted for obtained bactericidal effect of various concentrations of AgNPs@AV hybrids on E. coli

\subsection{Cytotoxicity}

In vitro cells study, Human Dermal Fibroblasts (HDF) cells were seeded into 96-well plates at a density of 104 in $100 \mu \mathrm{L}$ of medium per well and grown in RPMI 1640 cells medium supplemented with $10 \%$ NCBS, 100 units $\mathrm{mL}^{-1}$ penicillin and $0.1 \mathrm{mg} \mathrm{mL} \mathrm{m}^{-1}$ streptomycin under standard conditions in a conventional incubator with $5 \%$ $\mathrm{CO}_{2}$ at $37^{\circ} \mathrm{C}$ overnight. The cells were then treated with different concentrations of AgNPs@AV hybrids (10, $20,50,100,250$, and $500 \mu \mathrm{mol} \mathrm{mL}^{-1}$ ) respectively with $100 \mu \mathrm{L}$ per well and allowed for $36 \mathrm{hrs}$ of incubation. MTT assays at $490 \mathrm{~nm}$ using universal microplate spectrophotometer (BIO-TEK INSTRUMENTS.INC. USA)

\section{Results}

\subsection{Preparation and Characterization of AgNPs (a) AV hybrids}

Figure 2(a,b) shows the TEM image of as-synthesized AgNPs@AV hybrids, revealing that the NPs are predominantly spherical in the size range of $25 \mathrm{~nm}$. The 


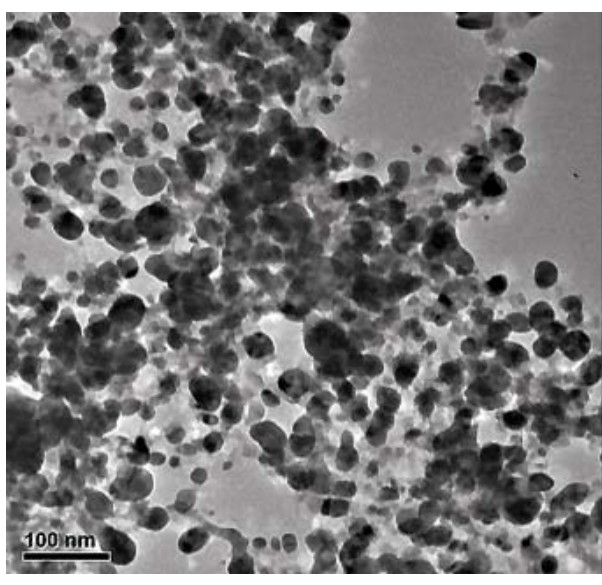

a

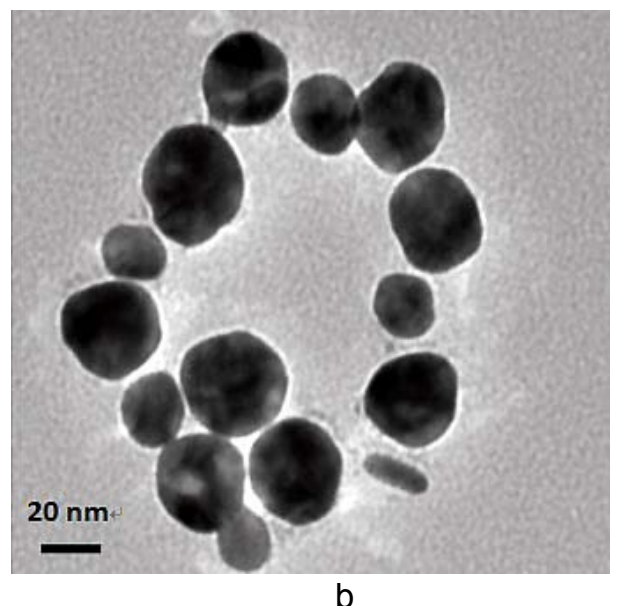

b

Figure 2 TEM image of AgNPs@AV: (a) lower magnification scopes, (b) At higher magnification

NPs could be well dispersed in water, and be stable for at least three months.

\subsection{X-ray diffraction (XRD) study}

The crystalline nature of Ag NPs was confirmed by the XRD analysis in Figure 3. The diffraction peaks at $\angle 38.03^{\circ}, \angle 46.18^{\circ}, \angle 64.6^{\circ}$ and $\angle 77.18^{\circ}$ correspond to the $\{111\},\{200\},\{220\}$ and $\{311\}$ facets [29] of the fcc crystal structure, respectively. The peak corresponding to the $\{200\}$ plane is more intense than the other planes, suggesting that the $\{200\}$ plane is the predominant orientation [30].

\subsection{Antibacterial effect}

Antibacterial tests were performed against the Gramnegative bacterium E. coli, on LB solid agar plates containing different concentrations of nanomaterials. Figure 4 shows the number of bacterial colonies grown on LB plates as a function of the concentration of NPs when approximately $105 \mathrm{CFU} \mathrm{mL}^{-1}$ were applied to the plates. Figure 4 displays the results of AgNPs@Aloe Vera hybrids, Ag NPs washed from Aloe Vera solution and Aloe Vera extract at different concentration of 10 , $50,100,200,500 \mu \mathrm{molL}^{-1}$. The first column is the control group. The data indicated that AgNPs@Aloe Vera hybrids have the highest antibacterial ability than the others at various doses tested. Moreover, the rate of inactivation by

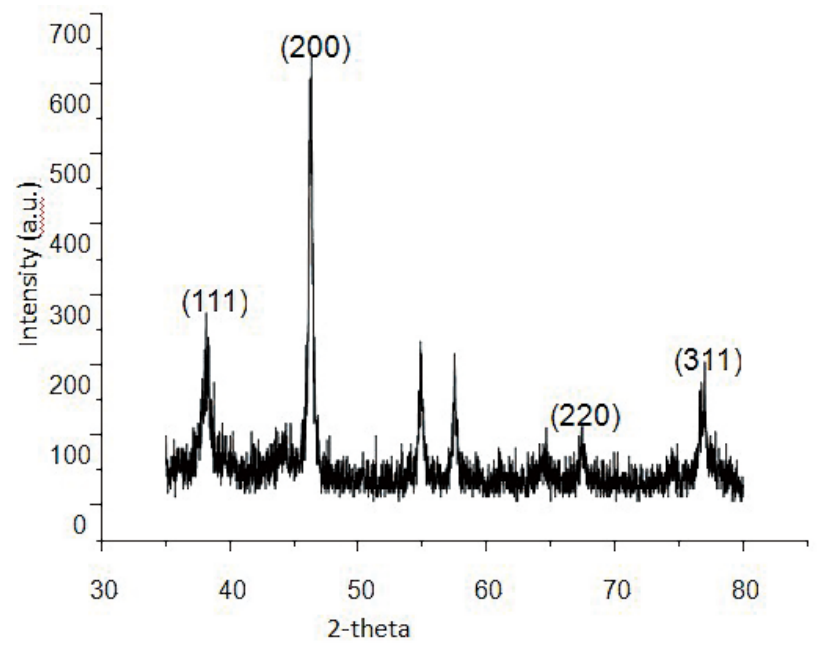

Figure 3 XRD pattern of AgNPs@Aloe Vera hybrids the composite varied proportionally with concentration. A concentration of $200 \mu \mathrm{molL}^{-1}$ caused $100 \%$ inhibition of bacterial growth.

\subsection{Cytotoxicity}

In vitro cells assays, HDF cells were exposed the different concentrations of AgNPs@Aloe Vera hybrids. The results of MTT assays show that AgNPs@Aloe Vera hybrids exhibit a significant cytotoxicity from 100 to 250 $\mu \mathrm{molL}^{-1}$ (see in Figure 5). In contrast,AgNPs@,Aloe Vera did not produce significant increase in cytotoxicity at the doses from 10 to $50 \mu \mathrm{molL}^{-1}$.

\section{Discussions}

AgNPs@AV hybrids were synthesized using the fresh Aloe Vera extract. TEM image revealed that the AgNPs@ AV hybrids were predominantly spherical with an average size of $25 \mathrm{~nm}$ in diameter, as shown in Figure 2. The crystalline nature of AgNPs@AV hybrids was confirmed by XRD study. We speculated that some active biological moleculars, benzoquinones in plant extracts might act as the reduction agents for formation of the NPs, as shown in Figure 6.

Large molecules enzymes and proteins in extract could control the size and shape of the NPs by weaker binding

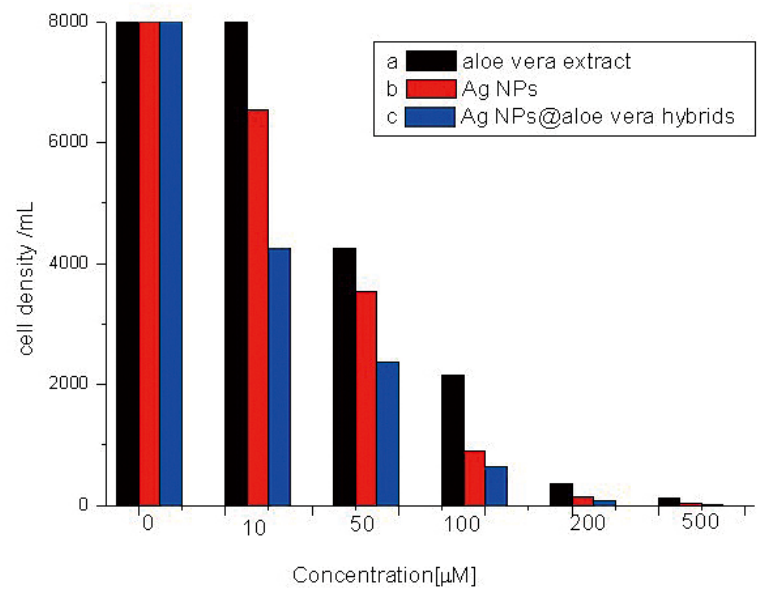

Figure 4 The antimicrobial activity comparation of different concentrations (a)Aloe Vera extract, (b) AgNPs washed from Vera extract, (c)Ag NPs@Aloe Vera hybrids 


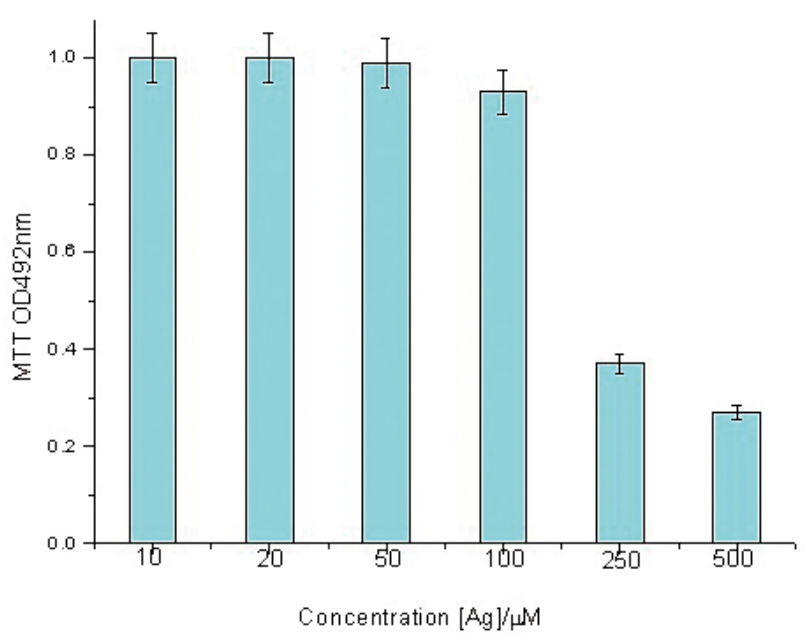

Figure 5 Dose-dependent cytotoxicity profiles of AgNPs@AV hybrids on HDF cells with nascent silver nanocrystals which lead to isotropic growth of the crystals and thus formation of spherical $\mathrm{Ag}$ NPs [31], which was displayed in Figure 7.

Ag NPs has been known to have effective inhibitory and antibacterial properties, as well as a broad range of antimicrobial activities, which has been used extensively to prevent and treat various diseases for centuries. Although the antibacterial mechanism is rather complex, many researches indicate Ag NPs appear to exert bacteriocidal activity predominantly through the release of silver ions followed (individually or in combination) by increasing membrane permeability, loss of the proton motive force, inducing de-energization of the cells and efflux of phosphate, leakage of cellular content, and disruption DNA replication [32]. The process was shown in Figure 8.

In light of our experimental results, we proposed a possible mechanism to interpret the combined antibacterial effects of AgNPs@AV hybrids to E. coli. From several aspects: First, the Ag nanostructure itself determines the strong antibacterial activities. Pal [33] et al. reported that both the nanoscale size and presence of a $\{111\}$ plane of Ag NPs to promote the biocidal

A B<smiles>O=C1c2cccc(O)c2C(=O)c2c(O)c(CO)cc(O)c21</smiles><smiles>Cc1cc(O)c2c(c1)C(=O)c1cccc(O)c1C2=O</smiles>

Aloeemodin antimicrobial<smiles>C=C=CC1c2c(O)cccc2C(O)c2cc(CO)cc(O)c21</smiles>

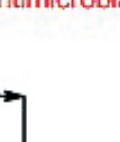

If Chrysophanol antimicrobial<smiles>Cc1cc(O)c2c(c1)C(=O)c1c(O)ccc(O)c1C2=O</smiles>

C

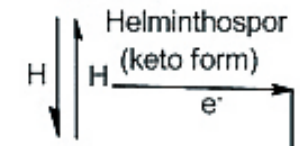

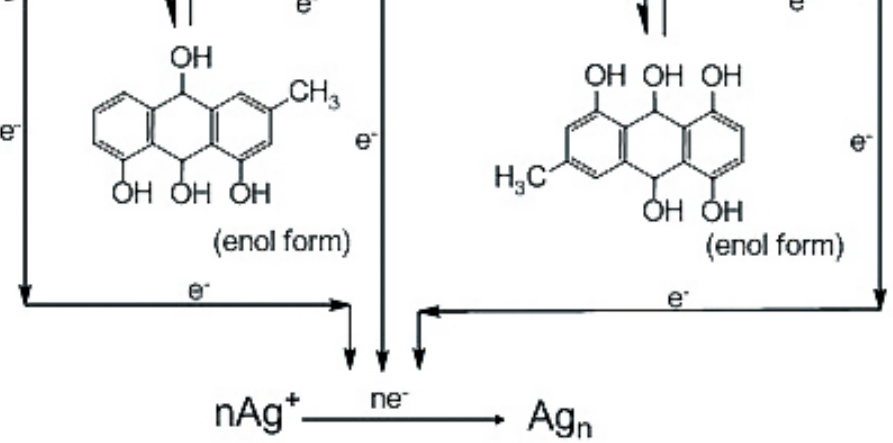

Figure 6 Possible mechanism of biosynthesis of Ag nanopariticles through quinine contained in fresh aloe vera extract

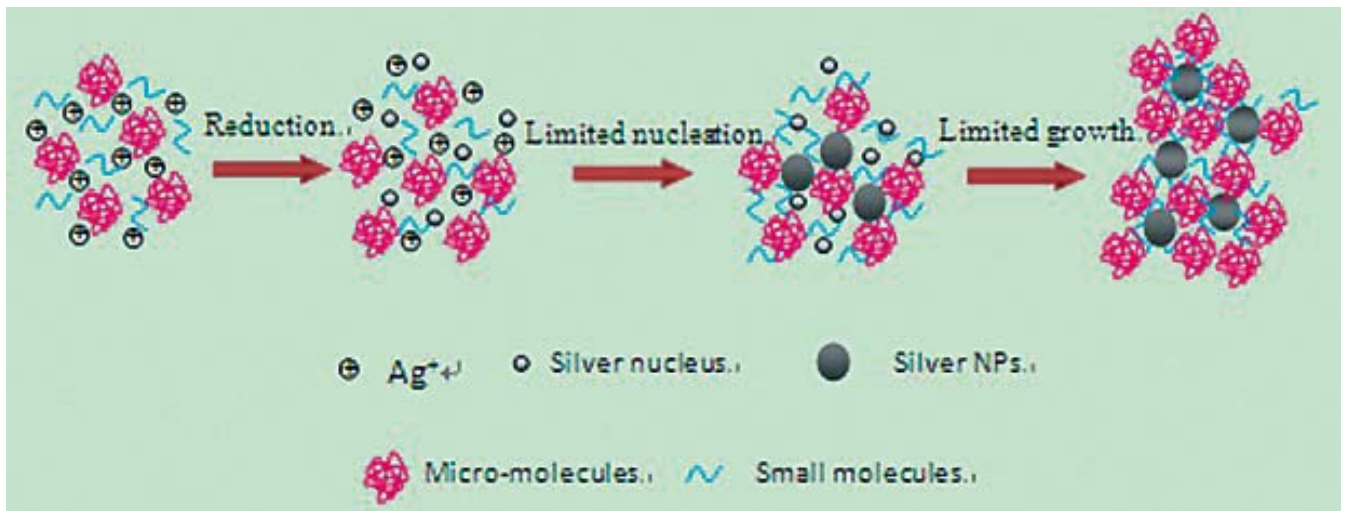

Figure 7 Schematic representation of the formation process of Ag NPs in aloe vera solution. A plenty of $\mathrm{Ag}^{+}$were added into the extract of aloe vera which contains many active small and large molecules. On one hand, the biological molecules might act as reducer to reduce $\mathrm{Ag}^{+}$to be $\mathrm{Ag} \mathrm{NPs}$; On the other hand, they were also responsible for the stabilization of resulting nanoparticles 

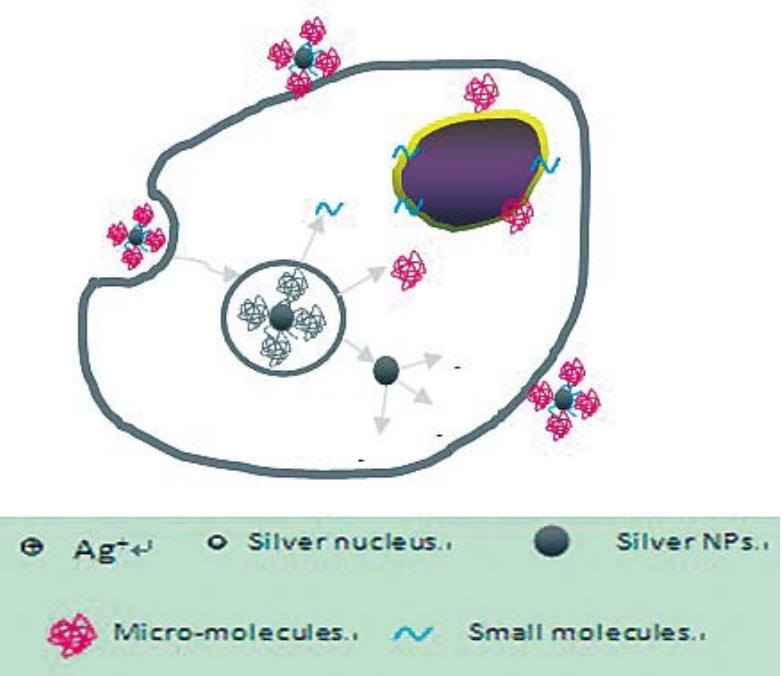

Figure 8 Possible synergistic antimicrobial mechanism of AgNPs@AV.

property on E. coli. In our XRD analysis, the $\{111\}$ planes are predominantly exposed faces of AgNPs@ AV hybrids. The main $\{111\}$ planes are responsible for the strong antibacterial effects. The outer membrane (OM) of E. coli consists of lipopolysaccharides (LPS), peptidoglycan, teichoic acids and s-layer proteins which render the cell surface negatively charged [34]. The NPs binding up to cells wall by $\mathrm{Ag}^{+}$. On one hand, $\mathrm{Ag}^{+}$could easily conjugate with cell membrane via electrostatic interactions so that impair the proper function of bacteria cell membranes. On the other hand, $\mathrm{Ag}^{+}$could also bind up to thiol-containing proteins by chelation, which greatly enhanced the membrane permeability [35].

Last but not least, Aloe Vera exhibited genotoxic on E. coli [36], but its capacity of piercing cellular wall was not as strong as NPs. Since the AgNPs@AV hybrids might contain some Aloe Vera active molecules around them, AgNPs@AV could act as "a carrier vehicle" to aid Aloe Vera active molecules transfer through cells wall to exert genotoxic actions to E. Coli leading to inactivation of the organisms $[35,36]$.

In a word, due to the complex constituents and structures involved in the AgNPs@AVhybrids, they showed synergistic effect on E. coli. This phenomenon could be well understood by "Drugs Cocktail Therapy" to cancer which has been proved to be effective to patients. The detailed mechanism needs to do further studies.

\section{Conclusions}

We describe a facile, sustainable, environment-friendly and low-cost process to prepare NPs by reduction of silver nitrate solution with Aloe Vera extract at room temperature. The experiment can be easily scaled up to industrial production. Besides being environmentally benign and good biocompatible of end-up products, we put up an innovative point which is to utilize the synergistic antibacterial effects by screening and combing different naturally-occurring medical plants extract and Ag NPs We speculate the synergistic activity is the same to other microorganisms. We believe that the novel green bactericides would be born from the plant extracts based nanotechnology.

\section{Acknowledgments}

This work is supported by the National Key Basic Research Program (973 Project) (2010CB933901), National 863 Hi-tech Project (2007AA022004, Important National Science \& Technology Specific Projects (2009ZX10004-311), National Natural Scientific Fund (No. 20771075 and No. 20803040), Special project for nanotechnology from Shanghai (No. 1052nm04100), New Century Excellent Talent of Ministry of Education of China (NCET-08-0350) and Shanghai Science and Technology Fund (10XD1406100).

\section{References}

1. Yang, D.; Cui, D., Advances and prospects of gold nanorods. Chem.Asian J. 2008, 3 (12), 2010-2022. doi:10.1002/asia.200800195

2. Panyam, J.; Labhasetwar, V., Biodegradable nanoparticles for drug and gene delivery to cells and tissue. Adv. Drug Delivery Rev. 2003, 55 (3), 329-347. doi:10.1016/S0169-409X(02)00228-4

3. Park, J.; Gu, L.; von Maltzahn, G.; Ruoslahti, E.; Bhatia, S.; Sailor, M., Biodegradable luminescent porous silicon nanoparticles for in vivo applications. Nat. Mater. 2009, 8 (4), 331-336. doi:10.1038/ nmat 2398

4. Uludag, Y.; Tothill, I., Development of a Sensitive Detection method of Cancer Biomarkers in Human Serum (75\%) using a Quartz Crystal Microbalance Sensor and Nanoparticles amplification system. Talanta 2010, 82, 277-282. doi:10.1016/j.talanta.2010.04.034

5. Sanpui, P.; Murugadoss, A.; Prasad, P.; Ghosh, S.; Chattopadhyay, A., The antibacterial properties of a novel chitosan-Ag-nanoparticle composite. Int. J. Food Microbiol. 2008, 124 (2), 142-146. doi:10. 1016/j.ijfoodmicro.2008.03.004

6. Cho, K.; Park, J.; Osaka, T.; Park, S., The study of antimicrobial activity and preservative effects of nanosilver ingredient. Electrochim. Acta 2005, 51 (5), 956-960. doi:10.1016/j.electacta.20 05.04.071

7. Kvitek, L.; Panac ek, A.; Soukupova, J.; Kolar, M.; Vec erova, R.; Prucek, R.; Holecova, M.; Zboril, R., Effect of surfactants and polymers on stability and antibacterial activity of silver nanoparticles (NPs). J. Phys. Chem. C 2008, 112 (15), 5825-5834. doi:10.1021/jp7 $11616 \mathrm{v}$

8. Stoimenov, P.; Klinger, R.; Marchin, G.; Klabunde, K., Metal oxide nanoparticles as bactericidal agents. Langmuir 2002, 18 (17), 66796686. doi:10.1021/la0202374

9. Xie, J.; Lee, J.; Wang, D.; Ting, Y., Silver nanoplates: from biological to biomimetic synthesis. ACS nano 2007, 1 (5), 429-439. doi:10.1021/ nn7000883

10.N, M.; Nadagouda; S, R.; Varma, Green synthesis of silver and palladium nanoparticles at room temperature using coffee and tea extract. Green Chem. 2008, 10 (8), 859-862. doi:10.1039/b804703k

11.Yang, D.; Chen, S.; Huang, P.; Wang, X.; Jiang, W.; Pandoli, O.; Cui, D., Bacteria-template synthesized silver microspheres with hollow and porous structures as excellent SERS substrate. Green Chem. 2010, 12, 2038-2042. doi:10.1039/c0gc00431f

12.Huang P, Kong Y, Li Z, Gao F, Cui D. Copper selenide nanosnakes: bovine serum albumin-assisted room temperature controllable synthesis and characterization. Nanoscale Res. Lett. 2010, 5(6), 949956. doi:10.1007/s11671-010-9587-0.

13.Huang P, Li Z, Hu H, Cui D. Synthesis and Characterization of Bovine Serum Albumin-conjugated Copper Sulfide Nanocomposites. J. Nanomater. 2010, doi:10.1155/2010/641545.

14.Huang P, Bao L, Yang D, Gao G, Lin J, Li Z, et al. Protein-directed solution-phase green synthesis of BSA-conjugated MxSey $(\mathrm{M}=\mathrm{Ag}$, $\mathrm{Cd}, \mathrm{Pb}, \mathrm{Cu}$ ) nanomaterials. Chem Asian J. In press.

15.Shankar, S.; Ahmad, A.; Pasricha, R.; Sastry, M., Bioreduction of chloroaurate ions by geranium leaves and its endophytic fungus yields gold nanoparticles of different shapes. J. Mater. Chem. 2003, 13 (7), 1822-1826. doi:10.1039/b303808b

16.Mukherjee, P.; Ahmad, A.; Mandal, D.; Senapati, S.; Sainkar, S.; Khan, M.; Parishcha, R.; Ajaykumar, P.; Alam, M.; Kumar, R., Fungus-mediated synthesis of silver nanoparticles and their immobilization in the mycelial matrix: a novel biological approach to 
29.Kannan, P.; John, S., Synthesis of mercaptothiadiazole-nctionalized gold nanoparticles and their self-assembly on Au substrates. Nanotechnology 2008, 19, 085602. doi:10.1088/0957-484/19/8/085 602

17.Kong, Y.; Chen, J.; Gao, F.; Li, W.; Xu, X.; Pandoli, O.; Yang, H.; Ji, J.; Cui, D., A Multifunctional Ribonuclease(C) $\backslash$ (C) $\backslash$ Conjugated CdTe Quantum Dot Cluster Nanosystem for Synchronous Cancer Imaging and Therapy. Small 2010, 6 (21), 2367-2373. doi:10.1002/ smll.201001050

18.Kong, Y.; Gao, F.; He, R.; Chen, J.; Xu, X.; Li, N.; Cui, D., Bioinspired synthesis of bovine serum albumin conjugated $\mathrm{Ag} 2 \mathrm{Se} / \mathrm{Se}$ core/ shell heterostructure nanoparticles at room temperature. Curr. Nanosci. $2010,6,446$.

19.Shankar, S.; Ahmad, A.; Sastry, M., Geranium leaf assisted biosynthesis of silver nanoparticles. Biotechnol. Prog. 2003, 19 (6), 16271631. doi:10.1021/bp034070w

20.Zawahry, M.; Hegazy, M.; Helal, M., Use of aloe iim treating leg ulcers and dermatoses. Int. J. Dermatol. 1973, 12 (1), 68-73. doi:10.11 11/j.1365-4362.1973.tb00215.x

21.Vogler, B.; Ernst, E., Aloe vera: a systematic review of its clinical effectiveness. Br. J. General Pract. 1999, 49 (447), 823.

22.Agarry, O.; Olaleye, M.; Bello-Michael, C., Comparative antimicrobial activities of aloe vera gel and leaf. Afr. J. Biotechnol. 2005, 4 (12), 1413-1414

23.CA, N.; LA, A.; D, P. J., Herbal Medicines: A guide for health-care for health-care professionals. 1996, 296.

24.Davis, R.; Donato, J.; Hartman, G.; Haas, R., Anti-inflammatory and wound healing activity of a growth substance in Aloe vera. J. Am. Podiatr. Med. Assoc. 1994, 84 (2), 77-81.

25.Vazquez, B.; Avila, G.; Segura, D.; Escalante, B., Antiinflammatory activity of extracts from Aloe Vera gel. J. Ethnopharmacol. 1996, 55, 69-75. doi:10.1016/S0378-8741(96)01476-6

26.Feng, Q.; Wu, J.; Chen, G.; Cui, F.; Kim, T.; Kim, J., A mechanistic study of the antibacterial effect of silver ions on Escherichia coli and Staphylococcus aureus. J. Biomed. Mater. Res., Part A 2000, 52 (4), 662-668. doi:10.1002/1097-4636(20001215)52:4<662::AIDJBM10>3.0.CO;2-3

27.Chandran, S.; Chaudhary, M.; Pasricha, R.; Ahmad, A.; Sastry, M., Synthesis of gold nanotriangles and silver nanoparticles using aloevera plant extract. Biotechnol. Prog. 2006, 22 (2), 577-583. doi:10.1021/bp 0501423

28.Philip, D., Biosynthesis of Au, Ag and Au-Ag nanoparticles using edible mushroom extract. Spectrochim. Acta, Part A 2009, 73 (2), 374381. doi:10.1016/j.saa.2009.02.037
30.Hart, L.; Van den Berg, A.; Kuis, L.; Van Dijk, H.; Labadie, R., An anti-complementary polysaccharide with immunological adjuvant activity from the leaf parenchyma gel of Aloe vera. Planta Med. 1989, 55 (6), 509-512.

31.Marambio-Jones, C.; Hoek, E., A review of the antibacterial effects of silver nanomaterials and potential implications for human health and the environment. J. Nanopart. Res. 2010, 12 (5), 1531-1551. doi:10.1007/s11051-010-9900-y

32.Pal, S.; Tak, Y.; Song, J., Does the antibacterial activity of silver nanoparticles depend on the shape of the nanoparticle? A study of the gram-negative bacterium Escherichia coli. Appl. Environ. microbiol. 2007, 73 (6), 1712-1720. doi:10.1128/AEM.02218-06

33.Fly, L.; Kiem, I., Tests of Aloe vera for antibiotic activity. Economic Botany 1963, 17 (1), 46-49. doi:10.1007/BF02985352

34.Paes-Leme, A.; Motta, E.; De Mattos, J.; Dantas, F.; Bezerra, R.; Caldeira-de-Araujo, A., Assessment of Aloe vera (L.) genotoxic potential on Escherichia coli and plasmid DNA. $J$ Ethnopharmacol. 2005, 102 (2), 197-201. doi:10.1016/j.jep.2005. 06.013

35.Gogoi, S.; Gopinath, P.; Paul, A.; Ramesh, A.; Ghosh, S.; Chattopadhyay, A., Green fluorescent protein-expressing Escherichia coli as a model system for investigating the antimicrobial activities of silver nanoparticles. Langmuir 2006, 22 (22), 9322-9328. doi:10.1021/la060661v

36.Morones, J. R.; Elechiguerra, J. L.; Camacho, A.; Holt, K.; Juan B Kouri4, The bactericidal effect of silver nanoparticles. $4 / 16 / 10 / 059$

Received 15 November, 2010; accepted 6 December, 2010; published online 16 December, 2010.

Copyright:(c) 2010 Y. Zhang et al. This is an open-access article License, which permits unrestricted use, distribution, and reproduction in any medium, provided the original author and source are credited. Nanotechnology 2005, 16 (10), 2346-2353. doi:10.1088/0957-448 distributed under the terms of the Creative Commons Attribution 